\title{
205. On a Non-linear Volterra Integral Equation with Singular Kernel
}

\author{
By Takashi KanAzAwA
}

(Comm. by Kinjirô KunugI, M. J. A., Sept. 13, 1971)

In the present paper we consider the solution $y(x)$ of the non-linear Volterra integral equation

$$
y(x)=f(x)+\int_{0}^{x} p(x, t) k(x, t, y(t)) d t
$$

where $p(x, t)$ is supposed to be unbounded in the region of integration.

Examples. $\quad p(x, t)=(x-t)^{-1 / 2}$, or $p(x, t)=t\left(x^{2}-t^{2}\right)^{-1 / 2}$.

Evans [1] studied a similar problem using the convolution. Our treatment below is more elementary than his. We also consider the continuity and differentiability with respect to a parameter of solutions of (1) when it contains a parameter.

1. Existence theorem. In equation (1) we shall assume the four conditions :

(a) $f(x)$ is continuous in the interval $I_{a}$,

$$
I_{a}=\{x \mid 0 \leqslant x \leqslant a\} \text {; }
$$

(b) $k(x, t, y)$ is continuous in the region $\Delta$,

where $\Delta=\{(x, t, y)|0 \leqslant t \leqslant x \leqslant a| y-,f(x) \mid \leqslant b\}$,

$$
\sup _{0 \leqslant t \leqslant x \leqslant a} k(x, t, f(x))=K,
$$

$k(x, t, y)$ satisfies a Lipschitz condition:

$$
\left|k\left(x, t, y_{1}\right)-k\left(x, t, y_{2}\right)\right| \leqslant L\left|y_{1}-y_{2}\right|
$$

(c) $\int_{0}^{x}|p(x, t)| d t \leqslant M<\infty \quad(0 \leqslant x \leqslant a)$;

(d) for any $\varepsilon>0$, there exists $\delta>0$, independent of $x$ and $\alpha$, such that

$$
\int_{\alpha}^{\alpha+\delta}|p(x, t)| d t<\varepsilon \quad \text { for all } 0 \leqslant \alpha \leqslant x-\delta .
$$

Theorem 1. Under the conditions (a), (b), (c), (d), equation (1) has a unique continuous solution on the interval $0 \leqslant x \leqslant h$, where $h$ is determind as follows:

for any $\rho, 0<\rho<1$, let $P=\min \left(\frac{\rho}{L}, \frac{b}{K}\right)$ and then let $h=\min (r, a)$, where $r$ is determined by

$$
\int_{0}^{x}|p(x, t)| d t \leqslant P \quad(0 \leqslant x \leqslant r) .
$$

Proof. For $n=1,2, \cdots$, let us put 
where $y_{0}(x)=f(x)$.

$$
y_{n}(x)=f(x)+\int_{0}^{x} p(x, t) k\left(x, t, y_{n-1}(t)\right) d t,
$$

Then, by our determination of $P$ and $h, y_{n}(x)$ is defined in $I_{h}$ and satisfies the inequality

By Lipschitz condition, for $x \in I_{h}$,

$$
\left|y_{n}(x)-f(x)\right| \leqslant b \quad(n=1,2, \cdots) .
$$

$$
\begin{aligned}
\left|y_{n+1}(x)-y_{n}(x)\right| & \leqslant L \int_{0}^{x}|p(x, t)|\left|y_{n}(t)-y_{n-1}(t)\right| d t \\
& \leqslant L \sup _{x \in I_{h}}\left|y_{n}(x)-y_{n-1}(x)\right| \int_{0}^{x}|p(x, t)| d t \\
& \leqslant L P \sup _{x \in I_{h}}\left|y_{n}(x)-y_{n-1}(x)\right| .
\end{aligned}
$$

Hence we have

$$
\left|y_{n+1}(x)-y_{n}(x)\right| \leqslant b(L P)^{n} \leqslant b \rho^{n} \quad(n=0,1,2, \cdots) .
$$

Since $0<\rho<1$, the sequence $\left\{y_{n}(x)\right\}$ is uniformly convergent in $I_{h}$ and $y(x)=\min _{n \rightarrow \infty} y_{n}(x)$ is a continuous solution of equation (1).

The uniqueness follows from the Lipschitz condition on $k(x, t, y)$.

2. Prolongation of solution.

Theorem 2. The solution curve of equation (1) in Theorem 1 can be prolonged to the terminal point $x=a$ of the interval $I_{a}$, when $k(x, t$, $y$ ) is defined and continuous in $0 \leqslant t \leqslant x \leqslant a,|y|<\infty$.

Proof. Let $y=\bar{y}(x)$ be the solution of (1) in the interval $I_{h}, 0 \leqslant x$ $\leqslant h(<a)$, where $h=\min (r, a)$ and $r$ is determined by

$$
\int_{0}^{x}|p(x, t)| d t \leqslant P=\frac{\rho}{L} \quad(0 \leqslant x \leqslant r) .
$$

Then, from Theorem 1 we know that the equation

$$
y(x)=f(x)+\int_{0}^{h} p(x, t) k(x, t, \bar{y}(t)) d t+\int_{h}^{x} p(x, t) k(x, t, y(t)) d t
$$

has a continuous solution $y=\overline{\bar{y}}(x)$ in the interval $h \leqslant x \leqslant 2 h(\leqslant a)$, because $\int_{0}^{h} p(x, t) k(x, t, \bar{y}(t)) d t$ is bounded and continuous.

The function

$$
y(x)= \begin{cases}\bar{y}(x) & 0 \leqslant x \leqslant h \\ \overline{\bar{y}}(x) & h \leqslant x \leqslant 2 h\end{cases}
$$

is the solution of (1) in the interval $I_{2 h}$.

Repeating the same procedure finite times, we can reach to $x=a$.

3. Equation containing a parameter. Let us consider the integral equation

$$
y(x)=f(x, \lambda)+\int_{0}^{x} p(x, t) k(x, t, y(t), \lambda) d t
$$

containing a parameter $\lambda$.

Theorem 3. In equation (2), we shall assume the following 
conditions :

$\left(\mathrm{a}^{\prime}\right) \quad f(x, \lambda)$ is continuous in the region $\{(x, \lambda)|0 \leqslant x \leqslant a,| \lambda \mid \leqslant l\} ;$

( $\left.\mathrm{b}^{\prime}\right) \quad k(x, t, y, \lambda)$ is bounded continuous in the region $\{(x, t, y, \lambda)|0 \leqslant t \leqslant x \leqslant a| y-,f(x, \lambda)|\leqslant b,| \lambda \mid \leqslant l\}$

and satisfies a Lipschitz condition $\left|k\left(x, t, y_{1}, \lambda\right)-k\left(x, t, y_{2}, \lambda\right)\right| \leqslant L\left|y_{1}-y_{2}\right| ;$

( c ) $\int_{0}^{x}|p(x, t)| d t \leqslant M<\infty \quad(0 \leqslant x \leqslant a)$;

( $\left.d^{\prime}\right)$ for any $\varepsilon>0$, there exists $a \delta>0$, independent of $\alpha$ and $x$, such that

$$
\int_{\alpha}^{\alpha+\tilde{o}}|p(x, t)| d t<\varepsilon \quad \text { for all } 0 \leqslant \alpha \leqslant x-\delta .
$$

Further suppose that for any $\lambda(|\lambda| \leqslant l)$ there exists a (unique) solution of (2) in the interval $I_{a}=\{x \mid 0 \leqslant x \leqslant a\}$.

Then the solution $y=y(x, \lambda)$ is continuous with respect to the parameter $\lambda$ in the region $\{(x, \lambda)|0 \leqslant x \leqslant a,| \lambda \mid \leqslant l\}$.

Proof. For any $\rho, 0<\rho<1$, let $h=\min (r, a)$, where $r$ is determined by $\int_{0}^{x}|p(x, t)| d t \leqslant \frac{\rho}{L}(0 \leqslant x \leqslant r)$.

First we consider the solution $y(x, \lambda)$ in the region $\{(x, \lambda) \mid 0 \leqslant x \leqslant h$, $|\lambda| \leqslant l\}$.

For any $\lambda+\Delta \lambda,|\lambda+\Delta \lambda|<1$, we have

$$
\begin{aligned}
y(x, \lambda+ & \Delta \lambda)-y(x, \lambda) \\
= & f(x, \lambda+\Delta \lambda)-f(x, \lambda) \\
& +\int_{0}^{x} p(x, t)\{k(x, t, y(t, \lambda+\Delta \lambda), \lambda+\Delta \lambda)-k(x, t, y(t, \lambda), \lambda+\Delta \lambda)\} d t \\
& +\int_{0}^{x} p(x, t)\{k(x, t, y(t, \lambda), \lambda+\Delta \lambda)-k(x, t, y(t, \lambda), \lambda)\} d t,
\end{aligned}
$$

and therefore

$$
\begin{aligned}
& |y(x, \lambda+\Delta \lambda)-y(x, \lambda)| \\
& \quad \leqslant \delta_{1}(\Delta \lambda)+L \int_{0}^{x}|p(x, t)||y(t, \lambda+\Delta \lambda)-y(t, \lambda)| d t+M \delta_{2}(\Delta \lambda),
\end{aligned}
$$

where $|f(x, \lambda+\Delta \lambda)-f(x, \lambda)| \leq \delta_{1}(\Delta \lambda)$,

$$
|k(x, t, y, \lambda+\Delta \lambda)-k(x, t, y, \lambda)| \leqslant \delta_{2}(\Delta \lambda) \text {. }
$$

Let

$$
\delta_{1}(\Delta \lambda)+M \delta_{2}(\Delta \lambda)=\delta(\Delta \lambda)
$$

then, from assumptions $\left(a^{\prime}\right)$ and $\left(b^{\prime}\right)$

Let

$$
\delta(\Delta \lambda) \rightarrow 0 \quad \text { as } \quad \Delta \lambda \rightarrow 0 .
$$

then from (3)

$$
\sup _{(x, \lambda) \in I_{h} \times A}|y(x, \lambda+\Delta \lambda)-y(x, \lambda)|=\delta(y),
$$

$$
\delta(y) \leqslant \delta(\Delta \lambda)+\rho \delta(y)
$$


or

$$
\delta(y) \leqslant \delta(\Delta \lambda) /(1-\rho) .
$$

Therefore, when $\Delta \lambda \rightarrow 0, \delta(\Delta \lambda) \rightarrow 0$ and $\delta(y) \rightarrow 0$, that is, $y(x, \lambda)$ is continuous with respect to $\lambda$.

Applying the same argument successively, we can prove the continuity of $y(x, \lambda)$ with respect to $\lambda$ on the whole interval $I_{a}$. Q.E.D.

From Theorem 3, we have evidently

Theorem 4. Suppose the equation (2) satisfies the following conditions :

( $\left.\mathrm{a}^{\prime \prime}\right) \quad f(x, \lambda), \frac{\partial}{\partial \lambda} f(x, \lambda)$ are continuous in the region

$$
\left\{(x, \lambda)|0 \leqslant x \leqslant a,| \lambda-\lambda_{0} \mid \leqslant l\right\} .
$$

$\left(\mathrm{b}^{\prime \prime}\right) \quad k(x, t, y, \lambda), \frac{\partial}{\partial y} k(x, t, y, \lambda), \frac{\partial}{\partial \lambda} k(x, t, y, \lambda)$

are continuous in the region

$$
\left\{(x, t, y, \lambda)|0 \leqslant x \leqslant t \leqslant a,| y-f(x, \lambda)|\leqslant b,| \lambda-\lambda_{0} \mid \leqslant l\right\} ;
$$

( c ) $\int_{0}^{x}|p(x, t)| d t \leqslant M<\infty \quad(0 \leqslant x \leqslant a)$

(d ) for any $\varepsilon>0$, there exists $a \delta>0$, independent of $\alpha, x, \lambda$, such that

$$
\int_{\alpha}^{\alpha+\delta}|p(x, t)| d t<\varepsilon \quad \text { for all } 0 \leqslant \alpha \leqslant x-\delta .
$$

Further suppose that for any $\lambda,\left|\lambda-\lambda_{0}\right| \leqslant l$, there exists a (unique) solution $y(x, \lambda)$ of $(2)$ in the interval $I_{a}$.

Then the solution $y(x, \lambda)$ is continuously differentiable with respect to $\lambda$ at $\lambda=\lambda_{0}$.

Acknowledgement. The author wishes to thank Professor M. Urabe for his kind guidance.

\section{Reference}

[1] G. C. Evans: Volterra's integral equation of the second kind with discontinuous kernel. Trans. Amer. Math. Soc., 11, 393-413 (1910). 